\section{Cureus}

Received 02/21/2019

Review began 02/21/2019

Review ended 02/27/2019

Published 02/28/2019

\section{(C) Copyright 2019}

Alhazmi et al. This is an open access article distributed under the terms of the Creative Commons Attribution License CC-BY 3.0., which permits unrestricted use, distribution, and reproduction in any medium, provided the original author and source are credited.

\title{
Efficacy and Safety of Catheter-directed Thrombolysis in Preventing Post- thrombotic Syndrome: A Meta-analysis
}

Luai Alhazmi $^{1}$, Abdelmoneim Moustafa ${ }^{2}$, Muhammad A. Mangi ${ }^{1}$, Ahmed Alamer ${ }^{3}$, Ehab Eltahawy ${ }^{1}$

1. Cardiology, University of Toledo Medical Center, Toledo, USA 2. Internal Medicine, University of Toledo Medical Center, Toledo, USA 3. Miscellaneous, University of Toledo Medical Center, Toledo, USA

$\square$ Corresponding author: Luai Alhazmi, luai.alhazmi@utoledo.edu Disclosures can be found in Additional Information at the end of the article

\section{Abstract}

Post-thrombotic syndrome (PTS) is a complication that can develop after deep vein thrombosis (DVT) of lower extremities. In this meta-analysis, we compare the different modalities for treatment of DVT in reducing the risk of PTS. The primary outcome was the risk of PTS, and the secondary outcome included the risk of bleeding events. Review Manager (version 5.3; Cochrane Collaboration software) was used to analyze the data that are represented as a forest plot. Meta-analysis indicated that catheter-directed thrombolysis (CDT) plus anticoagulation (AC) decreases the likelihood of developing PTS compared with the AC-only group with an odds ratio of 0.28 (0.12-0.64). A subgroup analysis of randomized control trial (RCT) studies was conducted, and findings suggest a slight decrease in the likelihood of PTS incidence in the $\mathrm{CDT}+\mathrm{AC}$ treatment group compared to the $\mathrm{AC}$ treatment group (odds ratio, $\mathrm{OR}=0.76 ; \mathrm{CI}=0.58$ 0.99). For the secondary outcome, a statistically significant increase in bleeding events in the intervention groups was reported with an OR of 3.38 (1.33-8.61), suggesting that the risk of bleeding was high in the CDT plus AC group. CDT in addition to conventional AC for patients with DVT decreases the likelihood of PTS development. The protective effect of CDT comes at the expense of an increase in bleeding risk by three-fold. The decision to utilize CDT to prevent PTS should be individualized according to patient risk factors for developing PTS and their risk of bleeding.

Categories: Cardiac/Thoracic/Vascular Surgery, Cardiology, Internal Medicine

Keywords: post-thrombotic syndrome, catheter directed thrombolysis, deep vein thrombosis

\section{Introduction}

Post-thrombotic syndrome (PTS) is a complication that can develop after deep vein thrombosis (DVT) of the lower extremities [1-3]. PTS can occur at various times after the initial episode but usually manifests within two years of initial DVT onset [3].

The clinical presentation of PTS varies in severity from mild, lower extremity swelling to significant complications such as chronic leg pain, venous claudication, and leg ulcerations. PTS is diagnosed primarily based on clinical presentation including signs and symptoms in patients with confirmed DVT. Current recommendations from the American Heart Association (AHA) for clinical diagnosis suggest waiting for acute DVT to subside and then utilizing clinical tools or scales such as the Villalta scale or the clinical, etiological, anatomic, pathophysiological (CEAP) classification among others [2]. PTS can affect the quality of life and functional status of affected patients, while also being a very costly health care burden [2].

How to cite this article

Alhazmi L, Moustafa A, Mangi M A, et al. (February 28, 2019) Efficacy and Safety of Catheter-directed Thrombolysis in Preventing Post-thrombotic Syndrome: A Meta-analysis. Cureus 11(2): e4152. DOI 10.7759 /cureus. 4152 
A combination of mechanisms contributes to PTS, including venous outflow obstruction, destruction of the venous valvular apparatus, development of venous reflux, calf muscle pump dysfunction, and reduced wall shear stress, which may trigger an inflammatory process within the affected vein. These mechanisms result in elevated venous pressures in the affected limb, particularly in an upright position, and can worsen venous reflux resulting in a vicious cycle of events [1].

Early and more complete thrombus clearance is believed by many physicians to relieve venous outflow obstruction, preserve valvular function, and reduce venous hypertension [3-

5]. Systemic thrombolysis showed significant reductions in residual thrombus and PTS development; however, it increased the risk of major bleeding by three folds compared to conventional anticoagulation therapy [6-7]. Consequently, the AHA guidelines recommended against the use of systemic thrombolysis in acute DVT [3]. Catheter-directed thrombolysis (CDT) is an alternative option for decreasing the incidence of PTS with reduction of bleeding events compared to systemic lytic therapy. There is a growing body of evidence supporting the role of CDT in achieving a significant reduction of PTS particularly in patients with DVT by restoring patency of the affected vein, minimizing valvular incompetence and venous hypertension in the early studies [8-9].

In this meta-analysis, $\mathrm{CDT}$ along with anticoagulation therapy $(\mathrm{CDT}+\mathrm{AC})$ is compared to anticoagulation (AC) therapy alone as treatment options for DVT. The subsequent risk reduction for PTS development following treatment is the primary outcome; the secondary outcome measure is the incidence of a bleeding incident (minor or major).

\section{Materials And Methods}

\section{Data sources and search strategy}

A systematic review was carried out in accordance with the preferred reporting items for systematic review and meta-analysis (PRISMA) guidelines [10]. An experienced, health sciences reference librarian performed the literature search. Using PubMed, Embase, Cochrane Central Register of Controlled Trials, and Web of Science, published literature was searched from the time frame of database inception until February 25, 2018. Using the options of advanced search, the website was thoroughly filtered for CDT or standard anticoagulation or iliofemoral/popliteal/lower-extremity DVT. To identify further articles, references were hand searched. All identified articles were compiled using Endnote.

\section{Study selection}

The eligibility of studies was assessed by two investigators (LA, AM), independently. Studies were included in the present analysis if they were original research (randomized control or case-control studies) and involved patients undergoing therapy for DVT. Therapies being compared must include $\mathrm{CDT}$ and AC or AC alone. Studies were included if they measured the outcome variables of interest for this analysis: PTS development and bleeding events.

Studies were excluded if they were case reports or review articles. Studies were also excluded if they did not report the outcomes of interest. Articles published in languages other than English were excluded, only if no translation was available.

\section{Data extraction and quality assessment}

Two reviewers (LA, AM) individually extracted data from studies that met the inclusion and exclusion criteria and compiled an electronic database. The extracted information included the year of publication, sample size, the mean age of the patients, the country in which the study 
took place, PTS diagnosis method, and duration of follow-up. Discrepancies in data extraction and assessing bias in individual studies were resolved through mutual discussion and consensus formulation. The modality for diagnosing PTS differed among included studies, reflecting the unstandardized diagnosis methods in clinical practice, subsequently, various methods, as reported by individual studies, were included in this analysis.

The quality of the selected studies was assessed by two reviewers (LA, HA) using the NewcastleOttawa Assessment Scale and a modified Jadad scale for non-randomized, observational studies, and randomized studies, respectively [11-12].

\section{Statistical analysis}

Review Manager (version 5.3; Cochrane Collaboration software; Copenhagen) was used for all statistical analysis. Forest plots, illustrating findings were generated. Treatment effects for both primary and secondary outcomes were analyzed using odds ratios, with a two-sided statistical significance level of 5\%. Summary statistics were assessed using odds ratios and the statistical heterogeneity of each plot was assessed using $\mathrm{I}^{2}$. The level of heterogeneity was demarcated as low $\left(\mathrm{I}^{2}=25 \%\right.$ to $\left.49 \%\right)$, moderate $\left(\mathrm{I}^{2}=50 \%\right.$ to $\left.74 \%\right)$, and high $\left(\mathrm{I}^{2}>75 \%\right)$ heterogeneity [13]. Forest plots were generated for all studies and subgroup analyses (randomized control trials [RCT], studies utilizing the Villata scale for diagnosing PTS).

\section{Results}

\section{Study selection and characteristics}

A total of 315 papers were identified out of which 126 were duplicates. After further screening, 42 articles were then assessed in full for eligibility. Only six studies were identified after the application of inclusion and exclusion criteria [2-7]. Some studies were excluded due to irrelevant outcomes compared to our meta-analysis [12-13]. The detailed literature search is highlighted in the PRISMA flow diagram (Figure 1). 


\section{Cureus}

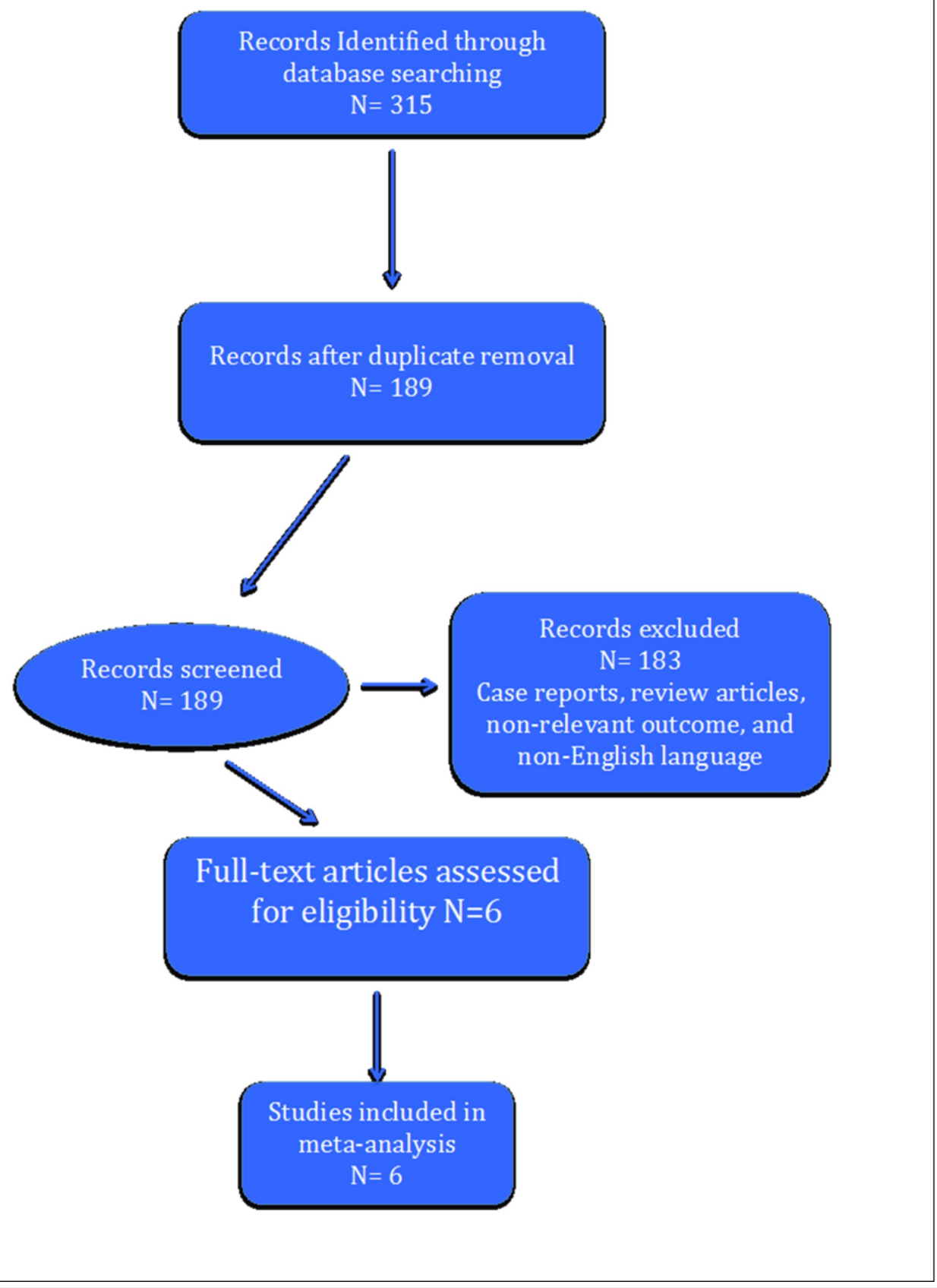

FIGURE 1: PRISMA flow diagram

The participant pool, of all six included studies totaling 1,077 patients, ranging in size from 50 to 692 patients with DVT. The duration of the follow-up ranged from six to 60 months. Two RCTs (CAVENT and ATTRACT trials) and four non-randomized, case-control studies included the experimental groups that received CDT therapy along with $\mathrm{AC}$ and control groups that received AC therapy alone, for acute lower extremity DVT [14-19]. Studies included multiple modes of PTS diagnosis including the Villata scale, CEAP classification, and clinical diagnosis based on signs and symptoms [14-15,17-19]. Table 1 outlines the baseline demographic and clinical characteristics of the studies included in analyses. 


\section{Cureus}

\begin{tabular}{|c|c|c|c|c|c|c|}
\hline Study & $\begin{array}{l}\text { Treatment } \\
\text { groups }\end{array}$ & $\begin{array}{l}\text { Sample } \\
\text { size (n) }\end{array}$ & $\begin{array}{l}\text { Mean age } \\
\text { (years) }\end{array}$ & $\begin{array}{l}\text { Follow-up } \\
\text { (months) }\end{array}$ & Country & $\begin{array}{l}\text { PTS } \\
\text { Definition }\end{array}$ \\
\hline AbuRahma et al. [14] & CDI/AC & $18 / 33$ & 50 & 55 & $\begin{array}{l}\text { United } \\
\text { States }\end{array}$ & $\begin{array}{l}\text { CEAP } \\
\text { classification }\end{array}$ \\
\hline Lee et al. [15] & CDT/AC & $27 / 26$ & 59 & 15 & Taiwan & Villalta scale \\
\hline Srinivas et al. [16] & CDT/AC & 27/28 & 53 & 6 & India & Villalta scale \\
\hline Ezelsoy et al. [17] & CDT/AC & $25 / 25$ & NA & 14 & Turkey & Clinically \\
\hline $\begin{array}{l}\text { Haig et al. [18] (CAVENT } \\
\text { trial) }\end{array}$ & CDT/AC & $87 / 89$ & 50 & 60 & Norway & Villalta scale \\
\hline $\begin{array}{l}\text { Vendantham et al. [19] } \\
\text { (ATTRACT trial) }\end{array}$ & CDT/AC & $337 / 355$ & 53 & 12 & $\begin{array}{l}\text { United } \\
\text { States }\end{array}$ & Villalta scale \\
\hline
\end{tabular}

\section{TABLE 1: Baseline characteristics}

PTS: post-thrombotic syndrome, CEAP: clinical, etiologic, anatomic, and pathophysiologic, CDT: catheter-directed therapy, AC: anticoagulation

${ }^{*}$ Clinical diagnosis based on sign and symptoms of PTS

Primary (incidence of PTS) and secondary (bleeding event) outcome data are shown in Table 2. PTS incidence was the primary outcome variable in all six included studies; only a single study did not include the secondary outcome variable of major or minor bleeding events [1419].

\begin{tabular}{|c|c|c|c|}
\hline Study & Group & PTS & Bleeding \\
\hline AbuRahma et al. [14] & $\mathrm{CDT}+\mathrm{AC} / \mathrm{AC}$ & $4 / 23$ & $5 / 5$ \\
\hline Lee et al. [15] & $\mathrm{CDT}+\mathrm{AC} / \mathrm{AC}$ & $5 / 13$ & $8 / 5$ \\
\hline Srinivas et al. [16] & $\mathrm{CDT}+\mathrm{AC} / \mathrm{AC}$ & $5 / 19$ & $4 / 0$ \\
\hline Ezelsoy et al. [17] & $\mathrm{CDT}+\mathrm{AC} / \mathrm{AC}$ & $7 / 14$ & NA/NA \\
\hline Haig et al. [18] (CAVENT trial) & $\mathrm{CDT}+\mathrm{AC} / \mathrm{AC}$ & $37 / 63$ & $20 / 0$ \\
\hline Vendantham et al. [19] (ATTRACT trial) & $\mathrm{CDT}+\mathrm{AC} / \mathrm{AC}$ & 157/171 & $46 / 38$ \\
\hline
\end{tabular}

\section{TABLE 2: Outcome variables: PTS and bleeding event results by study}

CDT: catheter-directed therapy, AC: anti-coagulation, PTS: post-thrombotic syndrome 


\section{Assessment of the quality of the included studies}

Quality assessment of the studies included in this meta-analysis showed all the studies to be of good quality. The observational studies being $9 / 9$ on the Newcastle Ottawa scale and the two

RCTs with a score of 5/5 on the Jadad scale [14-19].

\section{Primary outcome}

The first forest plot, of all six studies, assessed the treatment effect of CDT+AC therapy compared to AC alone as DVT treatments, in the likelihood of developing PTS. Results indicate that $\mathrm{CDT}+\mathrm{AC}$ as the therapy for DVT decreases the likelihood of developing PTS compared to AC therapy alone (odds ratio $[\mathrm{OR}]=0.28 ; 95 \%$ confidence intervals $[95 \% \mathrm{CI}]=0.12-0.64$; Figure 2). The heterogeneity of the included studies was high $\left(\mathrm{I}^{2}=83 \%\right)$.

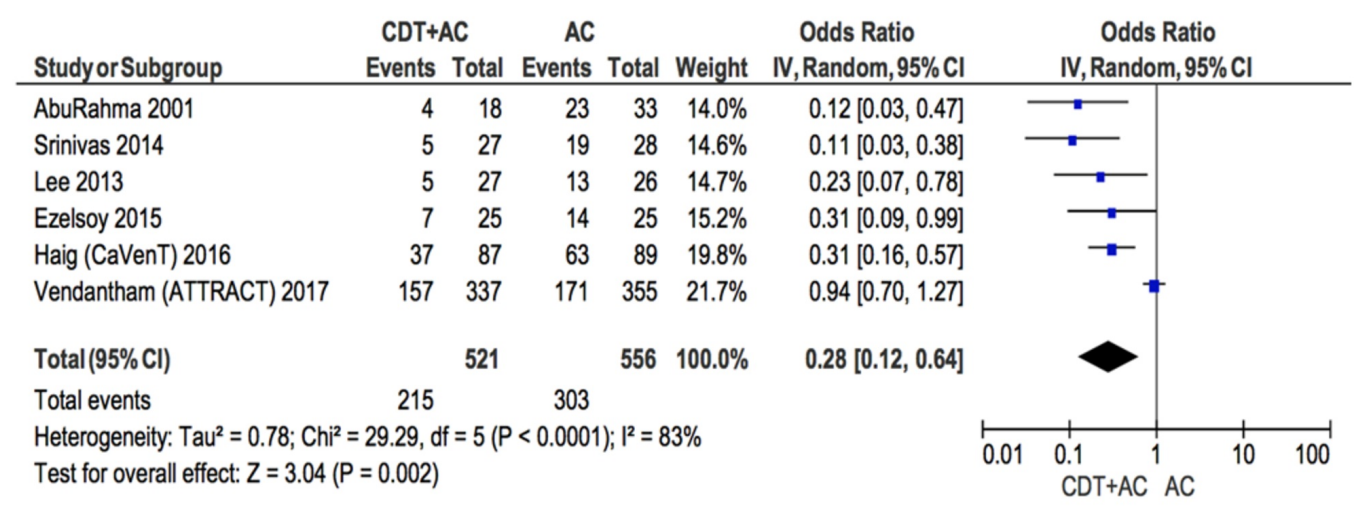

FIGURE 2: Forest plot comparing PTS incidence in CDT+AC vs AC

CDT: catheter-directed therapy, AC: anti-coagulation, PTS: post-thrombotic syndrome

The high level of heterogeneity suggested a need to explore sources of heterogeneity. An additional subgroup analysis removed the ATRRACT trial, which reduced the heterogeneity, was to $0 \%$.

\section{Randomized control trials subgroup analysis}

A subgroup analysis of RCT studies was conducted and findings suggest a slight decrease in the likelihood of PTS incidence in the CDT+AC treatment group compared to the AC treatment group $(\mathrm{OR}=0.76 ;[\mathrm{CI}]=0.58-0.99$; Figure 3$)[18-19]$. The heterogeneity of the included studies was high $\left(I^{2}=90 \%\right)$.

\begin{tabular}{|c|c|c|c|c|c|c|c|c|c|}
\hline Study or Subgroup & $\begin{array}{l}\text { CDT+A } \\
\text { Events }\end{array}$ & $\begin{array}{l}\text { AC } \\
\text { Total }\end{array}$ & $\begin{array}{r}\text { AC } \\
\text { Events }\end{array}$ & Total & Weight & $\begin{array}{c}\text { Odds Ratio } \\
\text { M-H,Fixed, } 95 \% \mathrm{Cl}\end{array}$ & \multicolumn{3}{|c|}{$\begin{array}{c}\text { Odds Ratio } \\
\text { M-H, Fixed, } 95 \% \mathrm{Cl}\end{array}$} \\
\hline Haig (CaVenT) 2016 & 37 & 87 & 63 & 89 & $28.7 \%$ & $0.31[0.16,0.57]$ & & - & \\
\hline Vendantham (ATTRACT) 2017 & 157 & 337 & 171 & 355 & $71.3 \%$ & $0.94[0.70,1.27]$ & & & \\
\hline Total $(95 \% \mathrm{Cl})$ & & 424 & & 444 & $100.0 \%$ & $0.76[0.58,0.99]$ & & 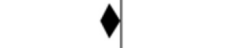 & \\
\hline Total events & 194 & & 234 & & & & & & \\
\hline $\begin{array}{l}\text { Heterogeneity: } \mathrm{Ch}^{2}=10.12, \mathrm{df}= \\
\text { Test for overall effect: } Z=2.05 \text { (F }\end{array}$ & $\begin{array}{l}1(P=0.00 \\
=0.04)\end{array}$ & $01) ; 1^{2}$ & $=90 \%$ & & & & 0.01 & $\begin{array}{lll}0.1 & 1 & 10 \\
C D T+A C & A C & \end{array}$ & 100 \\
\hline
\end{tabular}




\section{Cureus}

\section{FIGURE 3: Forest plot comparing PTS incidence in CDT+AC compared to AC in RCTs}

CDT: Catheter-directed therapy, AC: anti-coagulation, PTS: post-thrombotic syndrome, RCT: randomized control trial

Another subgroup analysis included only studies that used the Villalta scale to diagnose PTS (Figure 4) [15,17-19]. Findings suggest the likelihood of PTS development was lower in the group receiving $\mathrm{CDT}+\mathrm{AC}$ compared to the $\mathrm{AC}$ group $(\mathrm{OR}=0.65 ; \mathrm{CI}=0.51-0.84)$. The heterogeneity of the included studies was high $\left(\mathrm{I}^{2}=86 \%\right)$.

\begin{tabular}{|c|c|c|c|c|c|c|c|c|c|c|}
\hline \multirow{2}{*}{$\begin{array}{l}\text { Study or Subgroup } \\
\text { Lee } 2013\end{array}$} & \multicolumn{2}{|c|}{$\mathrm{CDT}+\mathrm{AC}$} & \multirow{2}{*}{$\begin{array}{r}\text { AC } \\
\text { Events } \\
13\end{array}$} & \multirow{2}{*}{$\begin{array}{r}\text { Total } \\
26\end{array}$} & \multirow{2}{*}{$\begin{array}{c}\text { Weight } \\
7.2 \%\end{array}$} & \multirow{2}{*}{$\begin{array}{c}\begin{array}{c}\text { Odds Ratio } \\
\text { M-H,Fixed, } 95 \% \mathrm{Cl}\end{array} \\
0.23[0.07,0.78]\end{array}$} & \multicolumn{4}{|c|}{$\begin{array}{c}\text { Odds Ratio } \\
\text { M-H,Fixed, } 95 \% \mathrm{Cl}\end{array}$} \\
\hline & 5 & 27 & & & & & & & & \\
\hline Srinivas 2014 & 5 & 27 & 19 & 28 & $10.1 \%$ & $0.11[0.03,0.38]$ & & & & \\
\hline Haig (CaVenT) 2016 & 37 & 87 & 63 & 89 & $23.7 \%$ & $0.31[0.16,0.57]$ & & $\rightarrow-$ & & \\
\hline Vendantham (ATTRACT) 2017 & 157 & 337 & 171 & 355 & $59.0 \%$ & $0.94[0.70,1.27]$ & & & & \\
\hline Total $(95 \% \mathrm{Cl})$ & & 478 & & 498 & $100.0 \%$ & $0.65[0.51,0.84]$ & & $\checkmark$ & & \\
\hline Total events & 204 & & 266 & & & & & & & \\
\hline $\begin{array}{l}\text { Heterogeneity: } \mathrm{Chi}^{2}=22.10, \mathrm{df} \\
\text { Test for overall effect: } Z=3.32\end{array}$ & $\begin{array}{l}3(P<0.0 \\
=0.0009\end{array}$ & 001); $1^{2}$ & $=86 \%$ & & & & 0.01 & $\begin{array}{l}0.1 \\
C D T+A C\end{array}$ & AC 10 & 100 \\
\hline
\end{tabular}

\section{FIGURE 4: Forest plot comparing PTS in CDT+AC vs. AC in} studies that used Villalta scale to define PTS

CDT: catheter-directed therapy, AC: anti-coagulation, PTS: post-thrombotic syndrome

\section{Secondary outcome}

Five of the included studies reported data on major and minor bleeding events. A subgroup analysis included only these five studies (Figure 5). An increased likelihood of bleeding events was found for participants who received $\mathrm{CDT}+\mathrm{AC}$ compared to those who received $\mathrm{AC}$ alone (OR $=1.95 ; \mathrm{CI}=1.34-2.84)$. However, no incidence of fatal or intracranial bleeding was reported. The heterogeneity of the included studies was moderate $\left(\mathrm{I}^{2}=62 \%\right)$.

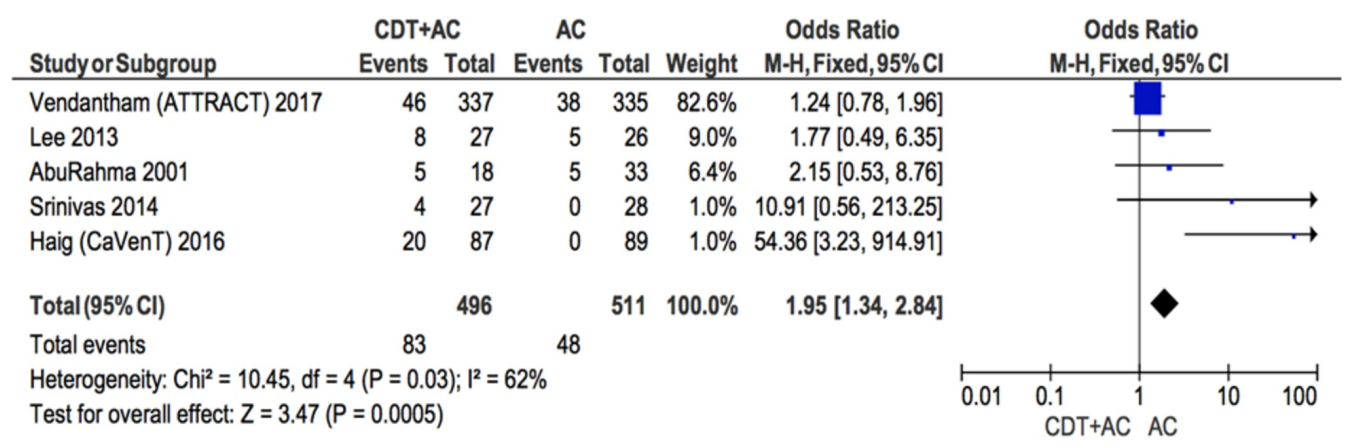

\section{FIGURE 5: Forest plot comparing the incidence of bleeding} events following $C D T+A C$ compared to $A C$ alone 


\section{Discussion}

The present meta-analysis investigated the treatment effects of $\mathrm{CDT}+\mathrm{AC}$ compared to $\mathrm{AC}$ therapy alone, in treating DVT. Specifically, the outcomes of interest were the incidence of the common long-term side effect, PTS as well as major or minor bleeding events. Findings of the analysis suggest that $\mathrm{CDT}+\mathrm{AC}$ reduced the risk of PTS compared to AC alone. Four of the six of the studies included in the present analysis were non-RCTs; these studies though relatively small in size indicated that $\mathrm{CDT}+\mathrm{AC}$ was effective in reducing PTS incidence compared to AC alone. The two RCTs included in the present analysis; the CAVENT trial and the ATTRACT trial were larger and designed to more accurately investigate the efficacy of CDT [17-18]. The CAVENT trial was a multicenter, randomized trial in which patients with DVT (Ilio-femoral and popliteal) were assigned to CDT and vitamin K antagonist (VKA) therapy versus VKA therapy. The ATTRACT trial was a multicenter, randomized trial in the United States that used pharmacomechanical catheter-directed thrombolysis (PCDT). The ATTRACT trial had the largest number of participants all studies included in this meta-analysis. The individual findings of this study suggest that there is no significant difference in the likelihood of either PTS development or the occurrence of bleeding events when comparing CDT+AC to AC alone. This differs from the pooled results, which show a decreased likelihood of PTS, this difference could be related to the differences in the follow-up duration of this large study in comparison to some of the smaller studies.

The current meta-analysis demonstrates decreased likelihood in PTS incidence in patients treated with $\mathrm{CDT}+\mathrm{AC}$ compared to anticoagulation alone. However, heterogeneity of the results of the primary outcome was high and that was predominantly driven by the ATTRACT trial results. The other potential source of heterogeneity between studies relates to differences in pharmacological therapy. Going into the details of these studies, AbuRahma, et al. used urokinase with loading dose 4,500 U/kg followed by infusion of 4,500 U/kg/hour for 24-48 hours [14]. Fibrinogen more than $100 \mathrm{mg} / \mathrm{dl}$ and activated partial thromboplastin time twice the control were made as the basis of the treatment. If fibrinogen was less than $100 \mathrm{mg} / \mathrm{dl}$, then urokinase was held for 6-12 hours and additional heparin was given at that time, but when fibrinogen was raised back to more than $100 \mathrm{mg} / \mathrm{dl}$, then urokinase resumed. Later, Lee, et al. also used the urokinase as a lytic agent [15]. Urokinase infused 600-1200 U/kg/hour over 48-72 hours with fibrinogen, and aPTT were maintained as the basis of the treatment. Moreover, in another study, streptokinase with a dose of 100,000 $\mathrm{U}$ was administered [16]. Two-thirds of the total dose given through catheter and one third through a sheath. The infusion was continued until a satisfactory result achieved. Unlike other studies in our meta-analysis, there was a manual aspiration of thrombus before infusion of lytic agent. Similar to this study, another study using the same mechanism of mechanical removal of thrombus followed by the use of alteplase as a lytic agent gained a successful result [17]. In the CAVENT trial, alteplase was used as a lytic agent with a dose of $0.01 \mathrm{mg} / \mathrm{kg} /$ hour, a maximum of $20 \mathrm{mg}$ in 24 hours. The infusion was continued until complete lysis or cease progression of a clot on venography with a maximum duration of 96 hours. In the ATTRACT trial, alteplase $<35 \mathrm{mg}$ was infused over 24-30 hours. Regarding the anticoagulation across the studies, coumadin was commonly used as anticoagulation, and goal INR was 2-3. Low molecular weight heparin and heparin were used as a bridge to anticoagulation. Given the nature of different drugs, dosing, and protocol of treatment, different outcomes are published, which created a huge heterogeneity in our metaanalysis.

In an attempt to overcome increased heterogeneity, ATTRACT trial was removed in subgroup analysis and the heterogeneity was reduced to $0 \%$. The level of heterogeneity in studies 
included in the meta-analysis indicate a high degree of difference between the studies, suggesting further investigation is warranted.

CDT remains controversial in preventing PTS. The current guidelines recommend AC alone for treatment of proximal DVT with level 2C recommendation [2]. An ongoing RCT (Dutch CAVA trial) comparing $\mathrm{CDT}$ to $\mathrm{AC}$ alone for treatment of proximal DVT may provide more clinical evidence for the benefit of CDT [20].

Regarding the major and minor bleeding events, the current meta-analysis revealed an increase in the likelihood of bleeding events following $\mathrm{CDT}+\mathrm{AC}$ therapy compared to AC alone. The major bleeding events included retroperitoneal, gastrointestinal, puncture-site bleeding that required a blood transfusion, bleeding that required surgical intervention, and minor bleeding events including puncture-site bleeding that does not require a blood transfusion, ecchymosis. The CAVENT trial demonstrated a high likelihood of bleeding events following CDT+AC therapy compared to AC therapy. The pooled result demonstrated an increased likelihood of bleeding events but was not as high as the CAVENT alone. This could be related to the difference in duration of follow-up between trials.

\section{Limitations}

The current meta-analysis had several limitations; four non-RCTs were compared to two RCTs; differences in these studies might influence the statistical results of pooled analyses. The heterogeneity of included studies was high and can mainly be attributed to the difference in size of studies including in our meta-analysis, mainly the ATTRACT trial, but may also be related to differences in follow-up duration.

\section{Conclusions}

CDT in addition to conventional anticoagulation for patients with DVT decreases the likelihood of PTS development. The protective effect of CDT comes at the expense of an increase in bleeding risk by three-fold. The decision to utilize CDT to prevent PTS should be individualized according to patient risk factors for developing PTS and their risk of bleeding.

\section{Additional Information}

\section{Disclosures}

Human subjects: All authors have confirmed that this study did not involve human participants or tissue. Animal subjects: All authors have confirmed that this study did not involve animal subjects or tissue. Conflicts of interest: In compliance with the ICMJE uniform disclosure form, all authors declare the following: Payment/services info: All authors have declared that no financial support was received from any organization for the submitted work. Financial relationships: All authors have declared that they have no financial relationships at present or within the previous three years with any organizations that might have an interest in the submitted work. Other relationships: All authors have declared that there are no other relationships or activities that could appear to have influenced the submitted work.

\section{References}

1. Ashrani AA, Heit JA: Incidence and cost burden of post-thrombotic syndrome . J Thromb Thrombolysis. 2009, 28:465-476. 10.1007/s11239-009-0309-3

2. Kahn SR, Shrier I, Julian JA, et al.: Determinants and time course of the postthrombotic syndrome after acute deep venous thrombosis. Ann Intern Med. 2008, 149:698-707. 10.7326/0003-4819-149-10-200811180-00004

3. Kahn SR, Comerota AJ, Cushman M, et al.: The post thrombotic syndrome: evidence-based 
prevention, diagnosis, and treatment strategies: a scientific statement from the American Heart Association. Circulation. 2015, 131:359. 10.1161/CIR.0000000000000185

4. Comerota AJ, Grewal N, Martinez JT, et al.: Post thrombotic morbidity correlates with residual thrombus following catheter-directed thrombolysis for iliofemoral deep vein thrombosis. J Vasc Surg. 2012, 55:768-773. 10.1016/j.jvs.2011.10.032

5. Vedantham S: Valvular dysfunction and venous obstruction in the post-thrombotic syndrome . Thromb Res. 2009, 123:62-65. 10.1016/S0049-3848(09)70146-X

6. Schweizer J1, Kirch W, Koch R, et al.: Short- and long-term results after thrombolytic treatment of deep venous thrombosis. J Am Coll Cardiol. 2000, 36:1336-1343. 10.1016/S07351097(00)00863-9

7. Goldhaber SZ, Buring JE, Lipnick RJ, Hennekens CH: Pooled analyses of randomized trials of streptokinase and heparin in phlebographically documented acute deep venous thrombosis. Am J Med. 1984, 76:393-397. 10.1016/0002-9343(84)90656-9

8. Elsharawy M, Elzayat E: Early results of thrombolysis vs anticoagulation in iliofemoral venous thrombosis. A randomised clinical trial. Eur J Vasc Endovasc Surg. 2002, 24:209-2014. 10.1053/ejvs.2002.1665

9. Bashir R, Zack CJ, Zhao H, Comerota AJ, Bove AA: Comparative outcomes of catheter-directed thrombolysis plus anticoagulation vs anticoagulation alone to treat lower-extremity proximal deep vein thrombosis. JAMA Intern Med. 2014, 174:1494-1501. 10.1001/jamainternmed.2014.3415

10. Liberati A, Altman DG, Tetzlaff J, et al.: The PRISMA statement for reporting systematic reviews and meta-analyses of studies that evaluate healthcare interventions: explanation and elaboration. BMJ. 2009, 339:2700. 10.1136/bmj.b2700

11. The Newcastle-Ottawa scale (NOS) for assessing the quality of nonrandomised studies in meta-analyses. (2015). Accessed: January 25, 2018: http://www.ohri.ca/programs/clinical_epidemiology/oxford.asp.

12. Oremus M, Wolfson C, Perrault A, Demers L, Momoli F, Moride Y: Interrater reliability of the modified Jadad quality scale for systematic reviews of Alzheimer's disease drug trials. Dement Geriatr Cogn Disord. 2001, 12:232-236. 10.1159/000051263

13. Zlowodzki M, Poolman RW, Kerkhoffs GM, et al.: How to interpret a meta-analysis and judge its value as a guide for clinical practice. Acta Orthop. 2007, 78:598-609. 10.1080/17453670710014284

14. AbuRahma AF, Perkins SE, Wulu JT, Ng HK: Iliofemoral deep vein thrombosis: conventional therapy versus lysis and percutaneous transluminal angioplasty and stenting. Ann Surg. 2001, 233:752-760.

15. Lee CY, Lai ST, Shih CC, Wu TC: Short-term results of catheter-directed intrathrombus thrombolysis versus anticoagulation in acute proximal deep vein thrombosis. J Chin Med Assoc. 2013, 76:265-270. 10.1016/j.jcma.2013.01.006

16. Srinivas BC, Patra S, Nagesh CM, Reddy B, Manjunath CN: Catheter-directed thrombolysis along with mechanical thromboaspiration versus anticoagulation alone in the management of lower limb deep venous thrombosis: a comparative study. Int J Angiol. 2014, 23:247-254. 10.1055/s-0034-1382157

17. Ezelsoy M, Turunc G, Bayram M: Early outcomes of pharmacomechanical thrombectomy in acute deep vein thrombosis patients. Heart Surg Forum. 2015, 18:222-225. 10.1532/hsf.1307

18. Haig Y, Enden T, Grøtta O, et al.: Post-thrombotic syndrome after catheter-directed thrombolysis for deep vein thrombosis (CaVenT): 5-year follow-up results of an open-label, randomised controlled trial. Lancet Haematol. 2016, 3:64-71. 10.1016/S2352-3026(15)00248-3

19. Vedantham S, Goldhaber SZ, Julian JA, et al.: Pharmacomechanical catheter-directed thrombolysis for deep-vein thrombosis. N Engl J Med. 2017, 377:2240-2252. 10.1056/NEJMoa1615066

20. DUTCH CAVA-trial: Catheter versus anticoagulation alone for acute primary (ilio)femoral DVT (NL28394). (2018). Accessed: January 5, 2018 : https://clinicaltrials.gov/ct2/show/NCT00970619. 